\title{
Parameter Determination Using 3D FIB-SEM Images for Development of Effective Model of Shale Gas Flow in Nanoscale Pore Clusters
}

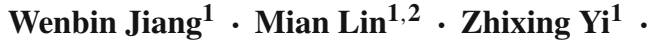 \\ Haishan $\mathrm{Li}^{1}$. Songtao $\mathrm{Wu}^{3}$
}

Received: 23 June 2016 / Accepted: 26 December 2016 / Published online: 31 December 2016

C Springer Science+Business Media Dordrecht 2016

\begin{abstract}
A large amount of nano-pores exists in pore clusters in shale gas reservoirs. In addition to the multiple transport regimes that occur on the nanoscale, the pore space is another major factor that significantly affects the shale gas recoverability. An investigation of the porescale shale gas flow is therefore important, and the results can be used to develop an effective cluster-scale pore network model for the convenient examination of the process efficiency. Focused ion beam scanning electron microscope imaging, which enables the acquisition of nanometre-resolution images that facilitate nano-pore identification, was used in conjunction with a high-precision pore network extraction algorithm to generate the equivalent pore network for the simulation of Darcy and shale gas flows through the pores. The characteristic parameters of the pores and the gas transport features were determined and analysed to obtain a deeper understanding of shale gas flow through nanoscale pore clusters, such as the importance of the throat flux-radius distribution and the variation of the tortuosity with pressure. The best parameter scheme for the proposed effective model of shale gas flow was selected out of three derived schemes based on the pore-scale prediction results. The model is applicable to pore-scale to cluster-scale shale gas flows and can be used to avoid the multiple-solution problems in the study of gas flows. It affords a foundation for further study to develop models for shale gas flows on larger scales.
\end{abstract}

Keywords Shale gas · Nanoscale pore cluster - Pore network model · FIB-SEM imaging · Apparent permeability

Mian Lin

linmian@imech.ac.cn

1 Institute of Mechanics, Chinese Academy of Sciences, Beijing 100190, China

2 University of Chinese Academy of Sciences, Beijing 100190, China

3 Research Institute of Petroleum Exploration \& Development, PetroChina, Beijing 100083, China 


\section{Introduction}

Shale gas is an unconventional natural gas trapped within shale formations. Its main content is methane, which is generated from the kerogen in shale through thermal evolution and is constrained in situ by the compactness and low permeability of shale. The source and reservoir of shale gas thus have the same location. Through high-pressure mercury penetration experiments and focused ion beam scanning electron microscope (FIB-SEM) imaging, a large amount of nano-pores has been observed in shale reservoirs. This feature is one of the main differences between shale gas reservoirs and conventional natural gas reservoirs. Hence, during recovery by hydraulic fracturing of the shale formation, the shale gas flows through two stages of channels consisting of nano-pore and micro-fracture networks (Akkutlu and Fathi 2012; Swami et al. 2013; Deng et al. 2014). The characteristics of the shale gas transport through the nano-pores significantly impact the gas recoverability (Lunati and Lee 2014) and have been a major research subject over the last few years.

One of the characteristics of nanoscale shale gas transport is the presence of multiple regimes that differ from the standard viscous flow. The Knudsen number is the key parameter used to distinguish different gas transport modes and is defined for flow through gas pores as the ratio of the mean free path of a gas molecule to the pore diameter. The dominant gas transport patterns in nano-pores are continuous flow (non-slip, $\mathrm{Kn}<10^{-3}$ ), slip flow $\left(10^{-3}<\mathrm{Kn}<10^{-1}\right)$, transition flow $\left(10^{-1}<\mathrm{Kn}<10\right)$ and Knudsen diffusion $(\mathrm{Kn}>10)$. Several studies have been conducted on gas flow processes in nano-capillaries and bundles of parallel nanotubes through theoretical analyses, experiments, and numerical simulations (Roy et al. 2003; Javadpour et al. 2007; Florence et al. 2007; Javadpour 2009; Civan 2010; Swami and Settari 2012; Fathi and Akkutlu 2012; Guo et al. 2013; Zhang et al. 2014). It has been observed that such gas flows disobey Darcy's law due to the existence of slip and other nonlinear behaviours. In addition, the derived apparent permeability of a rock compatible with the Darcy equation is not only dependent on the rock type, but also on the gas type, temperature, pressure, and other factors. Different equations of the gas flow flux and apparent permeability in a single nanotube have been proposed (Kazemi and Takbiri-Borujeni 2015; Harpreet and Javadpour 2016). The equation proposed by Javadpour (2009) considers the slip and Knudsen diffusion effect and is thus relatively complete and widely used (Darabi et al. 2012; Mehmani et al. 2013; Naraghi and Javadpour 2015).

Other important considerations are the effects of the pore space characteristics such as the pore size distribution, coordination number distribution, tortuosity, connectivity, and spatial configuration on the gas flow. There are two types of descriptions of these characteristics, namely macroscopic and microscopic descriptions. In a macroscopic description, the complicated pore networks (PNs) are substituted with bundles of parallel tubes to obtain an effective analytical relationship between the (apparent) permeability and several parameters of the pore structures. Civan $(2001,2002$, 2014) proposed a series of such models that describe more complicated pore structures. For the particular case of a shale gas reservoir, Darabi et al. (2012) proposed a framework function of the apparent permeability in which the pore surface fractal dimension $D_{f}$, tortuosity $\tau$, porosity $\Phi$, mean pore radius $R_{\text {avg }}$, and absolute permeability $K_{d}$ are used to describe the shale gas flow through the nano-pores. Because this approach is explicit and requires fewer inputs, it is always used for conceptual modelling and upscaling procedures (Naraghi and Javadpour 2015), with the model referred to as an "effective model". However, the approach involves the use of a framework function and several methods for determining the related parameters, resulting in the possibility of multiple specific forms of the model. There is thus the need to comparatively evaluate the different forms of the models to eliminate ambiguity and the multiple-solution problems. 
In the case of a microscopic description, which is also referred to as pore-scale modelling of shale gas flow when applied to the present subject of interest, the pore space is directly represented by 3D binary images or node-bond networks. The 3D binary images are obtained by FIB-SEM imaging or reconstruction of a 2D SEM image (Chen et al. 2013, 2015; Ma et al. 2014; Tahmasebi et al. 2015), and the PNs can be extracted from the 3D images or artificially generated using constraints. Mehmani et al. (2013) used local shrink and adjustment of the pore networks extracted from sandstone to approximate the micro- and nano-pore networks in shale and investigated the effect of the ratio of the nano-pores to the micro-pores on the apparent permeability using Javadpour (2009)'s flow flux formula. He found that the spatial configuration of the nano-pores significantly affected the gas permeability. Chen et al. (2013) used the lattice Boltzmann method (LBM) and FIB-SEM images of a shale sample to simulate Darcy flow and obtain the breakthrough curves. The non-Darcy flow was neglected because the Knudsen number was considered to be sufficiently small. Ma et al. (2014) extracted pore networks from reconstructed 3D images and, together with the similar non-Darcy flux formula, used them to examine the effect of TMAC (a parameter relates to slip effect) and the overall scaling of the throat size on the apparent permeability. Chen et al. (2015) performed LBM simulations using reconstructed 3D images of several shale samples and only calculated the absolute permeability and diffusivity on the pore scale. On the sample scale, a modified dusty gas model (DGM) that combines the effects of slippage and Knudsen diffusion were used to derive the correlation factor of the apparent permeability. However, the details of the pore-scale features were not provided. Tahmasebi et al. (2015) used a different method to reconstruct 3D images of shale samples from 2D images, with the objective of validating the reconstruction method using Darcy flow. The Avizo ${ }^{\circledR}$ software was used to predict the absolute permeability. The category of models used for microscopic description can be referred to as "pore-scale simulation-based models". The foregoing works that used approximated artificial or reconstructed networks or images emphasised the importance of considering the pore space characteristics when investigating shale gas flow.

Although the effective model approach is much easier to implement for larger models, it is difficult to determine the model parameters due to the possibility of the multiple-solution problems. This is primarily because the relationships between the model parameters and the pore-scale features are weak and indirect. Whereas a simulation-based model can be used to acquire the pore-scale flow features, previous relevant studies mainly focused on the overall behaviour of the shale gas flow in the shale sample or the effects of the model parameters.

The aim of the present study was to simulate the pore-scale shale gas flow through nanoscale pore clusters and attempt to establish a clear relationship between the apparent permeability and the parameters of the pore structure. To eliminate the uncertainties caused by the usage of artificially generated or reconstructed data, 3D FIB-SEM images of the shale sample were employed. This paper is divided into four parts. Section 2 presents a detailed description of the utilised data, especially those regarding the pore clusters, and the data preprocessing procedure. Section 3 introduces a new pore network model (PNM), including the extraction algorithm used to generate the pore networks from FIB-SEM images and the gas flow model for pore-scale simulation. In Sect. 4, the extracted pore networks and the related pore space and flow characteristics are presented and analysed. Finally, in Sect. 5, based on a combination of the pore-scale gas flow simulation results and the apparent permeability framework function, an effective model for nanoscale shale gas flow is proposed. The model was established by comparative evaluation of different parameter schemes. 


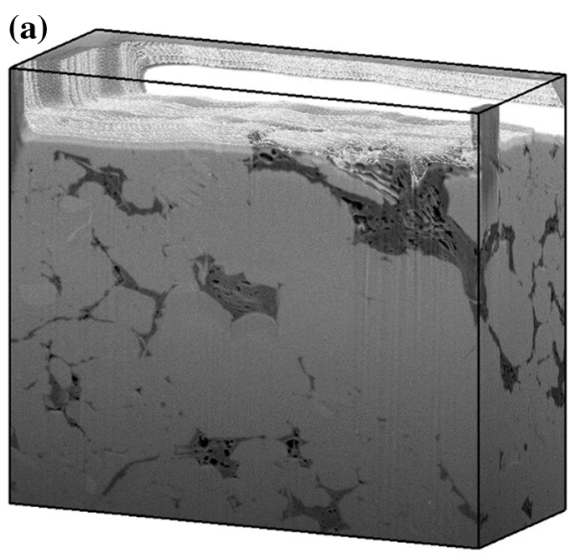

(c)

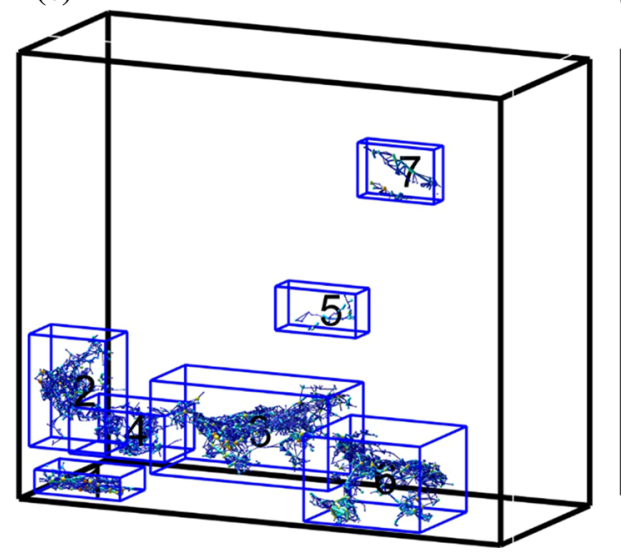

(b)

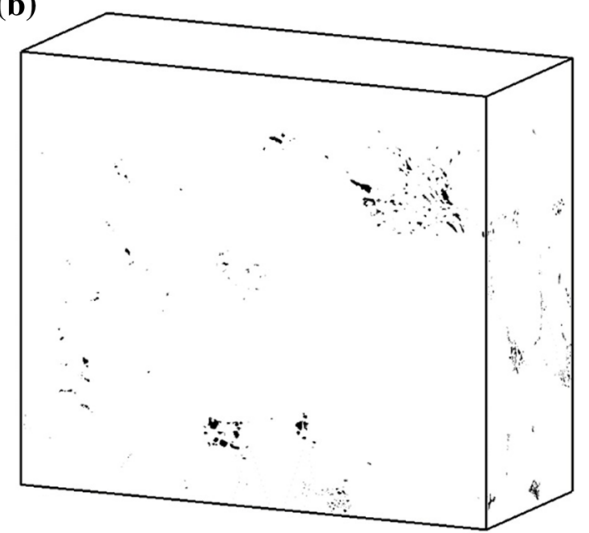

(d)

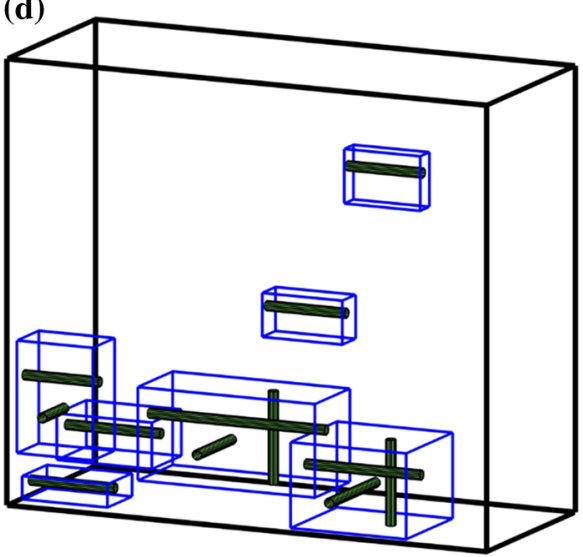

Fig. 1 FIB-SEM imaging data and processing results for the Sichuan marine shale. a FIB-SEM data, b void voxel distribution, $\mathbf{c}$ pore clusters and networks, $\mathbf{d}$ effective tube models

\section{Data Description}

FIB-SEM imaging is an advanced method for acquiring core imaging data with nanometre resolution. Through threshold segmentation of 3D images, the pore and matrix, for example, are separated and a binary dataset in which zero and one are used to represent the pore and matrix, respectively, is generated. This approach affords a direct description of the nano-scale pore space structure of shale.

FIB-SEM imaging data of grey-black mud shale obtained from the Longmaxi formation in Sichuan, China, was adopted in this study. The data were acquired at the laboratory of Research Institute of Petroleum Exploration \& Development, PetroChina. The shale was marine shale, buried at a depth of about $1319.2 \mathrm{~m}$ and with an Ro (vitrinite reflectance, an important parameter for estimating the thermal maturity) of 2.3 and TOC of $3.6 \%$ (Fig. 1a). The voxel numbers of the entire dataset were $1024 \times 884 \times 406$, and the minimum voxel size was $10 \mathrm{~nm}$. In the obtained 3D images, light grey indicated the matrix, which mainly comprised quartzes and clay minerals; dark grey indicated kerogen; and deep black indicated voids. The kerogen was dispersed through the matrix. The area ratio occupied by the kerogen 
in some parts was large, and there were also numerous connected void voxels. A threshold of 35 was set for the segmentation of the voids and solid using the Kapur-Sahoo-Wong (Maximum Entropy) thresholding method of the ImageJ software (Schneider et al. 2012); voxels with grey values lower than 35 were considered voids, while those with values equal to or higher than 35 were considered solids. The 3D binary dataset was then generated (Fig. 1b).

From the FIB-SEM images, the pore spaces were determined to consist of several isolated sub-spaces. A sub-space is an aggregation of interconnected void voxels that is isolated from other sub-spaces. Each sub-space, namely a pore cluster, consists of a series of connected pores and pore throats. Every pore cluster can be identified and labelled using the bwlabeln function in MATLAB ${ }^{\circledR}$. The pore clusters may be connected by hydraulic fractures (Chen et al. 2013). An understanding of shale gas transport through pore clusters is thus critical to the evaluation of shale gas recovery.

Pore clusters are of varying sizes. To avoid property fluctuation due to microscale spatial heterogeneity, the considered domain size of a pore cluster should be sufficiently large. Pore clusters with larger domain sizes are more likely to be connected by the generated fractures. Chen et al. (2013) conducted an sREV (statistical representative elementary volume) analysis of marine shale FIB-SEM images and found that the minimum dimension of an sREV in any direction was about $960 \mathrm{~nm}$. Consequently, in the present study, the minimum dimension of a pore cluster in the flowing directions was set to $1600 \mathrm{~nm}$, which is quite reasonable. Seven pore cluster domains were selected, as shown in Fig. 1c, and identified as S1-S7. The X, Y, Z anisotropies of each of the pore clusters were considered, effectively resulting in a total of 21 clusters. These 21 clusters were identified using the format $\mathrm{S}^{* *}$, where the second character is a number between 1 and 7 (indicating the pore cluster index) and the third character is $\mathrm{X}$, $\mathrm{Y}$, or Z (indicating the flow direction). However, there were some clusters with dimensions in a flow direction less than the minimum dimension. Such clusters were eliminated. This left 12 pore clusters, which were used as the objects for investigating shale gas flow through nano-pores.

\section{Pore Network Model: AB Model}

The numerical models used for flow simulation are of two main categories. Those of the first category directly discretise the pore spaces into lattices or grids, and the LBM or NavierStokes equations are used. Those of the second category are referred to as PNMs, in which the pore spaces are represented by a pore network consisting of a series of pores and pore throats, as illustrated in Fig. 2. The throats are the only flow channels, while the pores constitute the storage spaces. The computation efficiency of the former is much lower than that of the latter. Take LBM as an example, in order to ensure computation accuracy, there must be 4-5 lattices in a nanometre pore throat generally. The number of lattices for a whole sample is huge, and the computation time is too long. Finite difference method (FDM) even refers to grid mesh generation problem of complex geometry boundaries. In PNM, the problem is represented with linear or nonlinear equations that are much easier to solve with numerical methods.

Models of the first category used in previous studies only considered the Darcy and partial non-Darcy effects (Fathi and Akkutlu 2012; Chen et al. 2015). Pore-scale modelling of shale gas flow with LBM is still conducted in a capillary (Zhang et al. 2014) and is incipient when applied to 3D complex porous spaces. In contrast, a PNM easily incorporates the existing flow flux formula of the pore throat and also considers the complete non-Darcy effects. It is thus more promising for investigating the effects of the pore space characteristics on shale gas flow when used in conjunction with real shale pore networks. A PNM has other advantages 


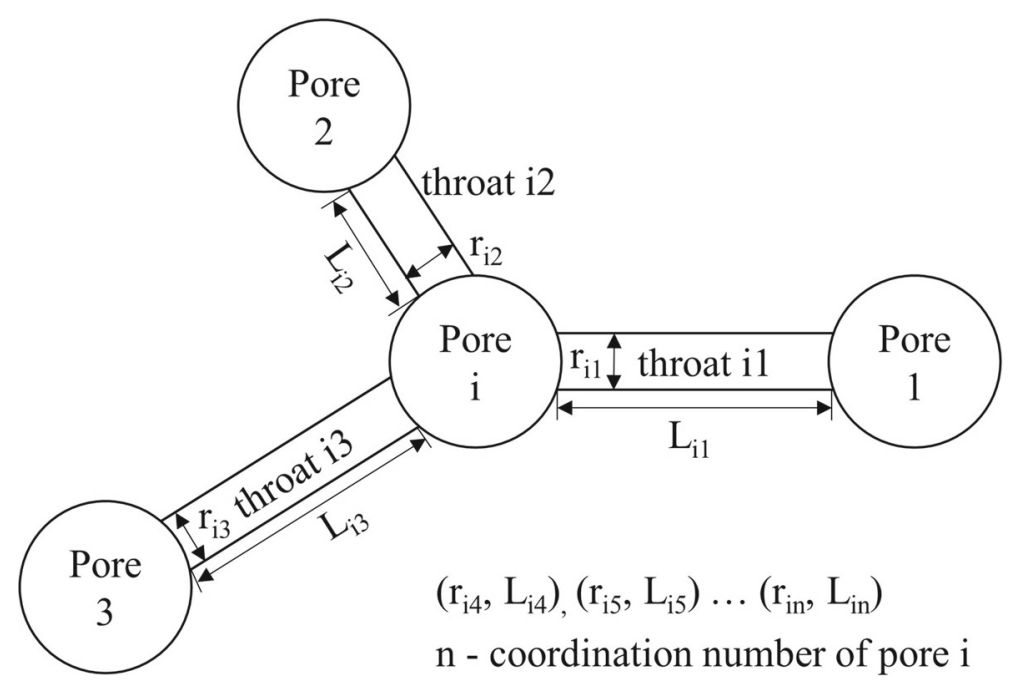

Fig. 2 Pore body and pore throat parameters

over image-based models (such as LBM, FDM), namely its containing information about the pores and throats during their development, and the non-requirement of information for identification with other morphological models. However, the difficulty of using a PNM is the need to ensure that the results of the calculations based on the extracted pore network are consistent with reality. In other words, the extraction of the pore network should be accurate.

\subsection{Pore Network Extraction: AB Algorithm}

We used a newly developed pore network extraction algorithm, the $\mathrm{AB}$ (axis \& ball) algorithm (Yi et al. under review), to extract the pore networks from the shale samples. The basic principle of the $\mathrm{AB}$ algorithm is the constraining of the maximal balls on the centrally located medial axis. The algorithm combines the advantages of the media axis (Lindquist et al. 1996; Jiang et al. 2007) and maximal ball (Dong and Blunt 2009) approaches and consists of six steps: (1) building the inscribed spheres; (2) identifying the medial axis; (3) constraining the maximal balls (MBs) on the medial axis; (4) defining the pores and pore throats; (5) segmenting the pores and pore throats; and (6) calculating the parameters of the pores and pore throats. The flow chart is shown in Fig. 3. Several detailed modifications and improvements of the algorithm ensure that the predicted petrophysical properties, the definitions of the elements, and the network connectivity are physically realistic. The new features include (1) utilisation of the inscribed spheres to better centre the axis and guarantee that every throat is located at a hydraulic restriction; (2) use of a dual-speed expansion algorithm to segment the pore spaces and ensure that the pores occupy most of the pore spaces and that every single void voxel can be assigned to a pore block or throat block; and (3) utilisation of an equivalent throat length formula that eliminates the use of adjustable coefficients to calibrate the absolute permeability and enhance the stability and adaptability. The $\mathrm{AB}$ algorithm was verified using 13 samples that included sand packs, sandstones, and carbonates, and the average relative errors of the absolute permeability predictions were $3.50 \%$ (Yi et al. under review). 


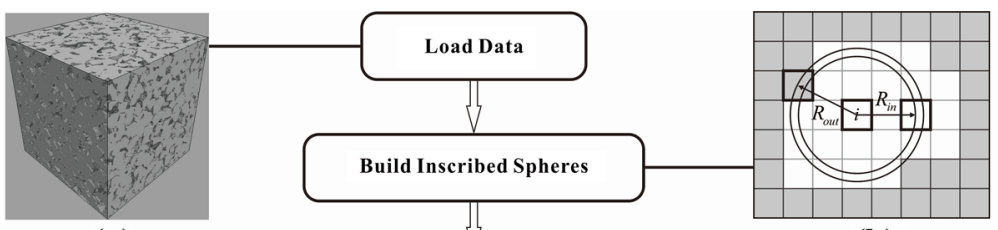

(a)

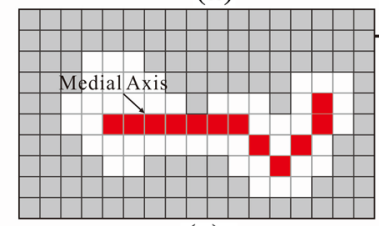

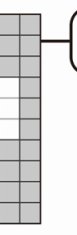
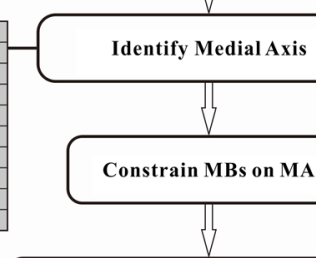

(b) (c)

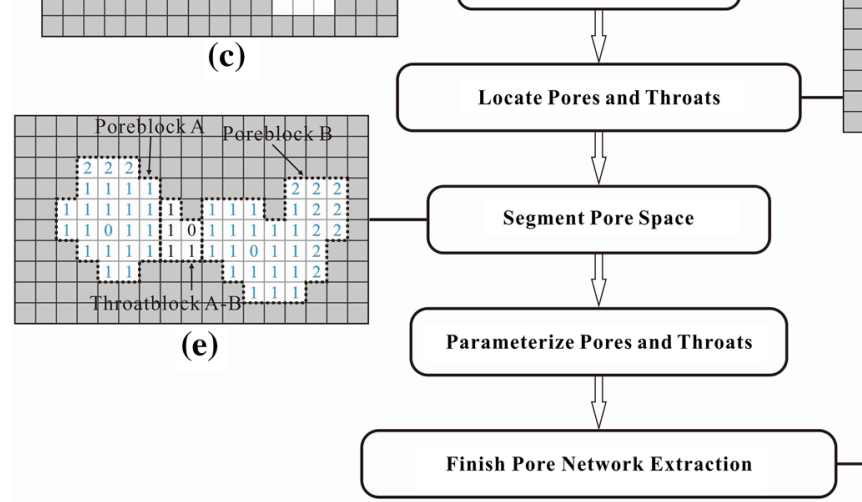

(e)

Legend:

Fig. 3 Flow chart of $\mathrm{AB}$ algorithm

\subsection{Absolute Permeability Calculation}

After the construction of the pore networks, the absolute permeability was calculated with the assumption of the existence of only viscous flow in the throats. Based on Darcy's law, the absolute permeability is given by

$$
K_{d_{\mathrm{AB}}}=\frac{Q \mu L}{A \rho_{w} \Delta p}
$$

where $L$ is the length of the sample in the pressure gradient direction, $A$ is the cross-sectional area of the sample, $\Delta p$ is the pressure difference between the inlet and outlet ends of the sample, $\rho_{w}$ is the density of water, $\mu$ is the viscosity of water, and $Q$ is the average flux of the pores at the inlet and outlet ends of the ample. The fluxes were determined by solving the linear equations of the flux conservation of all the pores in the network using Gauss-Seidel iteration. Considering pore $i$ as an example, the corresponding flux conservation equation according to the Hagen-Poiseuille law is as follows:

$$
\sum_{j=1}^{n} Q_{i j}=\sum_{j=1}^{n}-\pi \frac{r_{i j}^{4} \rho_{w}}{8 \mu}\left(\frac{p_{i}-p_{j}}{L_{i j}}\right)
$$

where $Q_{i j}$ is the flux of the throat between pore $i$ and pore $j\left(\mathrm{~kg} / \mathrm{m}^{2} / \mathrm{s}\right), n$ is the number of throats linked to pore $i$ (also known as the coordination number), $p_{i}$ and $p_{j}$ are the pressures 
in the two pores $(\mathrm{Pa})$, and $r_{i j}$ and $L_{i j}$ are, respectively, the radius and length of the throat between the two pores (m), as illustrated in Fig. 2.

\subsection{Apparent Permeability Calculation}

The pore networks extracted by the $\mathrm{AB}$ algorithm were used to simulate the shale gas flow. In addition to viscous flow, there were other flow regimes in the throats, such as slippage and Knudsen diffusion. A gas flux equation that considered these flow regimes and the effect of the pore surface roughness was applied to all the throats of the pore network (Darabi et al. 2012). By combining terms with the same order, the formula can be expressed as follows:

$$
\left\{\begin{array}{l}
Q_{i j}=B_{i j}\left(p_{i}-p_{j}\right)+C_{i j}\left(p_{i}^{2}-p_{j}^{2}\right) \\
B_{i j}=-\frac{2 \pi r_{i j}^{3} M}{3 \times 10^{3} R T L_{i j}}\left(\frac{8 \times 10^{3} R T}{\pi M}\right)^{0.5}\left(\frac{d_{m}}{2 r_{i j}}\right)^{D_{f}-2}-\frac{M \pi r_{i j}^{3}}{8 \times 10^{3} R T L_{i j}}\left(\frac{8 \times 10^{3} \pi R T}{M}\right)^{0.5}\left(\frac{2}{\alpha}-1\right) \\
C_{i j}=-\frac{\pi r_{i j}^{4}}{8 \mu} \frac{M}{2 \times 10^{3} R T L_{i j}}
\end{array}\right.
$$

In accordance with the flux conservation law, the above flow relationship was applied to all the throats. Consequently, the nonlinear equations, with the pressures of all the pores as unknown variables, can be expressed as follows:

$$
\left.\left.\sum_{j} Q_{i j}=\sum_{j} B_{i j}\left(p_{i}-p_{j}\right)\right)+\sum_{j} C_{i j}\left(p_{i}^{2}-p_{j}^{2}\right)\right)=0
$$

where $M$ is the gas molar mass (set to $16 \mathrm{Kg} / \mathrm{Kmol}$ for methane), $R$ is the gas constant $(8.31441 \mathrm{~J} / \mathrm{mol} / \mathrm{K}), T$ is the temperature (set to $400 \mathrm{~K}), d_{m}$ is the diameter of a gas molecule ( $3.8 \times 10^{-10} \mathrm{~m}$ for methane), $\mu_{g}$ is the viscosity of gas $\left(1.210^{-5} \mathrm{~Pa} \mathrm{~s}\right)$, and $\alpha$ is the tangential momentum accommodation coefficient (TMAC, a dimensionless parameter related to the gas, set to 0.8; Agrawal and Prabhu 2008; Javadpour 2009). The pore surface fractal dimension $D_{f}$ was specifically introduced into Eq. (3) to reflect the effect of the pore surface roughness on the Knudsen diffusion (Coppens 1999) and was determined by the 3D cubic covering method (CCM; Ai et al. 2014). The nonlinear Eq. (4) were solved using the Newton-Raphson iterative method to determine the pressures in all the pores.

The apparent permeability $K_{\text {app }}$ is given by

$$
K_{\text {app }}=\frac{Q_{g} \mu_{g} L}{A \rho_{g} \Delta p}
$$

where $Q_{g}$ is the average flux at the inlet and outlet of the sample, and $\rho_{g}$ is the density of gas $\left(\mathrm{Kg} / \mathrm{m}^{3}\right)$, which can be calculated with respect to the temperature and pressure using the equation of state for an ideal gas, $\rho_{g}=\frac{p M}{10^{3} R T}$. $K_{\text {app }}$ has a similar definition as the absolute permeability, except that, unlike the latter, which only depends on the pore structure, the former also varies with the pressure and temperature, because the parameters $B_{i j}$ and $C_{i j}$ in Eq. (3) depend on $p$ and $T$.

\section{Results Obtained by AB Model}

This section discusses the results of the application of the AB model to the above-mentioned 12 samples, to afford an insight into pore-scale shale gas flow. 
Table 1 Parameters from AB model

\begin{tabular}{lrrlrrllllr}
\hline Sample & $D_{f}$ & $\phi_{e}(\%)$ & $\phi_{f}(\%)$ & $N_{p}$ & \multicolumn{1}{c}{$N_{t}$} & $C N_{\mathrm{avg}}$ & $R_{\mathrm{a} . \mathrm{avg}}(\mathrm{nm})$ & $\tau$ & $K_{d_{\mathrm{AB}}}(\mathrm{nD})$ & $K_{d_{\mathrm{LBM}}}(\mathrm{nD})$ \\
\hline S1X & 2.54 & 10.87 & 0.67 & 154 & 298 & 2.45 & 11.08 & 1.62 & 1445.39 & 1132.92 \\
S2X & 2.40 & 3.62 & 0.24 & 380 & 913 & 2.44 & 9.00 & 2.47 & 32.19 & 26.15 \\
S2Y & 2.40 & 3.62 & 0.28 & 380 & 929 & 2.60 & 8.98 & 2.07 & 14.51 & 18.17 \\
S3X & 2.40 & 3.25 & 0.25 & 873 & 2235 & 2.77 & 9.12 & 1.65 & 35.79 & 32.61 \\
S3Y & 2.40 & 3.25 & 0.27 & 852 & 2302 & 2.89 & 9.17 & 2.42 & 7.38 & 6.15 \\
S3Z & 2.40 & 3.25 & 0.26 & 850 & 2330 & 2.86 & 9.17 & 2.68 & 13.61 & 12.51 \\
S4X & 2.44 & 3.92 & 0.27 & 410 & 892 & 2.60 & 8.57 & 2.05 & 17.47 & 15.70 \\
S5X & 2.14 & 1.61 & 0.05 & 25 & 30 & 1.44 & 10.18 & 1.32 & 18.58 & 14.70 \\
S6X & 2.34 & 3.79 & 0.26 & 586 & 1542 & 2.68 & 10.11 & 1.99 & 121.47 & 128.65 \\
S6Y & 2.34 & 3.79 & 0.28 & 651 & 1540 & 2.59 & 10.12 & 2.19 & 85.99 & 108.68 \\
S6Z & 2.34 & 3.79 & 0.26 & 617 & 1544 & 2.54 & 10.02 & 2.65 & 4.97 & 4.45 \\
S7X & 2.26 & 4.22 & 0.15 & 41 & 62 & 1.93 & 13.42 & 1.41 & 330.97 & 381.67 \\
\hline
\end{tabular}

$N_{p}$ and $N_{t}$ are the numbers of pores and throats in the pore network, respectively. $C N_{\text {avg }}$ is the average coordination number. $R_{\mathrm{a} \text { avg }}$ is the arithmetic average throat radius. $K_{d_{\mathrm{AB}}}$ and $K_{d_{\mathrm{LBM}}}$ are, respectively, the absolute permeability values from the AB model and LBM.

\subsection{Absolute Permeability Verification}

The accuracy of the extracted pore networks is the basis of the following analysis, and the absolute permeability is used as a judgment index. Because of the very small domain size of the clusters and the ultra-low absolute permeabilities, which foreclose experimental verification, the results of the LBM two-relaxation-time (TRT) model are used as reference. Pan et al. (2006) evaluated different lattice Boltzmann schemes used for the simulation of porous media and found that the TRT model could overcome the viscosity dependence of the computed permeability (Okabe and Blunt 2004, with results that are consistent with those of the analysis of ideal samples. Table 1 presents the absolute permeability results obtained using the pore networks extracted by the $\mathrm{AB}$ algorithm. As can be seen, $K_{d}$ varies between $4 n D$ and $1132 n D$. The average relative error between the AB algorithm and LBM results is $6.56 \%$, which is quite reasonable. The good consistency between the $\mathrm{AB}$ algorithm and LBM indicates that the pore networks extracted by the $\mathrm{AB}$ algorithm are equivalent to the actual pore spaces. This lays a good foundation for the following discussion related to the non-Darcy flow.

\subsection{Apparent Permeability}

When slippage and Knudsen diffusion are taken into consideration, the apparent permeability varies with the pressure. The apparent permeabilities of all the samples were calculated for eight different average pore pressures, namely, $0.1,1,2,5,8,10,20$, and $40 \mathrm{MPa}$, with the temperature fixed at $400 \mathrm{~K}$. Figure 4 shows the variation with pressure of the non-dimensional apparent permeability $\lambda$, which is defined as the ratio of $K_{\text {app }}$ to $K_{d}$. The value of $\lambda$ increases rapidly with decreasing pressure. At a pressure of $40 \mathrm{MPa}, \lambda$ is close to 1 , while it is as large as 31-88 (depending on the sample) when the pressure is $0.1 \mathrm{MPa}$. It can be seen from the enlarged plots in Fig. 4 that S5X is relatively large, attributable to its small $D_{f}$, which implies that the Knudsen diffusion is minimally inhibited by the pore surface roughness. S2X, S2Y, 


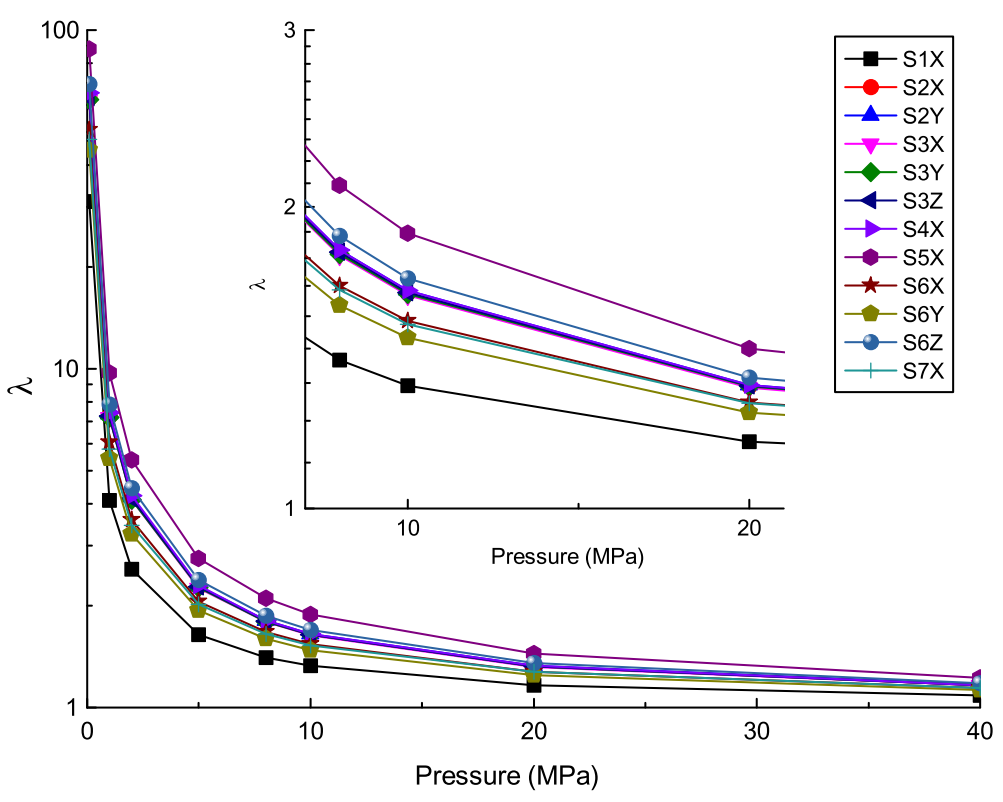

Fig. 4 Variation of the non-dimensional apparent permeability with the pressure

S3X, S3Y, S3Z, and S4X are comparable. The Df values of the S6 clusters are larger than those of the S5 clusters but smaller than those of the others. The exception is S6Z the value of $\lambda$ of which is larger than those of S6X and S6Y. The reason for this will be discussed later.

\subsection{Characteristic Parameters of Pore Clusters}

Figure 5 shows the pore networks extracted by the AB algorithm. It is evident that $\mathrm{S} 1-$ S4 and S6 have more developed pore networks, with the other clusters having fewer pores and throats. The coordination number distribution, throat number-radius distribution, and throat flux-radius distribution determined from the statistics of the extracted pore network parameters are also presented in Fig. 5.

Porosity Porosity is the ratio of the void volume of a material to the total volume. In porous media, not all the voids are connected to the inlet and outlet ends, but there are also some inner isolated voids. Moreover, not all the connected voids contribute to the fluid flow. There are therefore different special definitions of porosity. For example, flowing porosity $\phi_{f}$ is the ratio of the void volume that the fluid flows through to the total volume. In a PNM, it is the ratio of the volume of the throats with flux magnitudes larger than 0 to the total volume. $\phi_{f}$ is different from the effective porosity $\phi_{e}$, which is the ratio of the volume of the connected voids to the total volume.

Table 1 lists the $\phi_{e}$ and $\phi_{f}$ values for all the samples. As can be seen, the $\phi_{e}$ values are within $1.61-10.87 \%$, while the $\phi_{f}$ values are within $0.05-0.67 \%$. The ratios of $\phi_{f}$ to $\phi_{e}$ are within $3.11-8.31 \%$, which indicates that some voids do not contribute to flow. Because the flow paths in the different directions are different, the flow porosities in the different directions are also different. In the case of S3, the flow porosities in the $\mathrm{X}, \mathrm{Y}$, and $\mathrm{Z}$ directions varied within $0.25-0.27 \%$. 


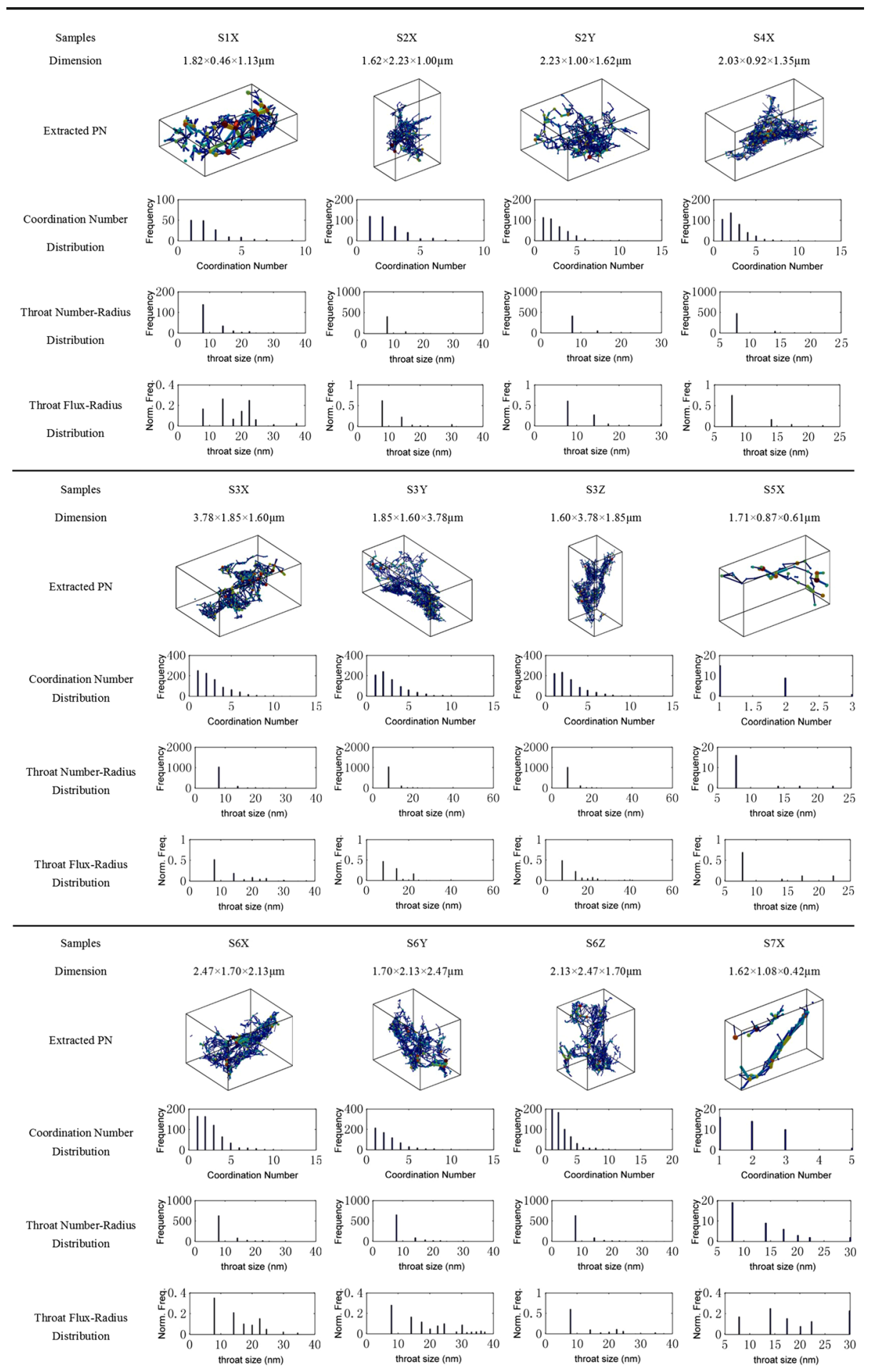

Fig. 5 Results obtained by the AB model 
Coordination Number Distribution The coordination number $(\mathrm{CN})$ of a pore is the number of throats connected to the pore. The five most developed pore clusters in this study were observed to have more complex connectivities, and their coordination numbers could exceed 10. In addition, the number of pores was found to decrease exponentially with increasing coordination number. However, S5 and S7 were less developed, with their number of pores decreasing linearly with the coordination number. From Table 1, it can be seen that the main coordination numbers of all the samples are 1 or 2 . The fact that the pores with $\mathrm{CN}=1$ are most predominant indicates the presence of a large number of pores that are blind ends to gas flow and that the nano-pores in shale significantly differ from those in sandstone, which has an average coordination number of 4 .

Throat Number-Radius Distribution Figure 5 shows that all the samples predominantly contain throats with radii less than $10 \mathrm{~nm}$ and that the number of throats with a given radius decreases with increasing value of the radius. The maximum radius is less than $40 \mathrm{~nm}$, which is less than ten times the minimum radius. The arithmetic mean throat radius is between 8.57 and $13.42 \mathrm{~nm}$, more precisely about $10 \mathrm{~nm}$.

Throat Flux-Radius Distribution Unlike the throat number-radius distribution, which is an intrinsic feature of a pore space, the throat flux-radius distribution is determined by the flow behaviour. It is determined by dividing the range of the throat radii into several sub-bins. The flux magnitudes of the throats with radii within the same bin are then summed and divided by the total flux of all the throats. The relationship between the normalised summed flux and the representative throat radius of the corresponding bin is subsequently determined. The results obtained in this study for Darcy flow are shown in Fig. 5, based on which the 12 samples can be divided into two categories. Category 1 includes S2X, S2Y, S3X, S3Y, S3Z, S4X, $\mathrm{S} 5 \mathrm{X}$, and S6Z, for which the absolute permeability is smaller than $100 n D$ (Table 1) and the contribution of the throats with radii less than $10 \mathrm{~nm}$ to the total flux is greater than $50 \%$, indicating that the contribution of the small throats cannot be neglected. Category 2 includes S1X, S6X, S6Y, and S7X. The absolute permeabilities of these samples are at least $100 \mathrm{nD}$, which is at least one order larger than those of the Category 1 samples, and the contribution of the throats with radii larger than $10 \mathrm{~nm}$ to the total flux is greater than $50 \%$. It is thus obvious that, although throats with radii smaller than $10 \mathrm{~nm}$ are much more than those with larger radii, the contribution of the smaller throats to the flux is a quite different issue.

Moreover, samples of the same pore cluster but with different directions may belong to different categories. Take pore cluster S6 as an example, a comparison of the arithmetic average throat radii and throat radius distributions in the three directions reveals that they are respectively very close; however, the absolute permeability in the $\mathrm{Z}$ direction is two orders smaller than that in the other two directions. Therefore, using only the average throat radius determined by the algorithm to estimate the absolute permeability of the samples may produce large discrepancies. The specific paths of the flows should be taken into consideration.

As can be seen from Fig. 4, the $\lambda$ curves for S6X and S6Y belong to Category 1 and are very similar, while that for S6Z belongs to Category 2 and is obviously smaller. The main reason for this is that the throats of the Category 2 samples with radii larger than $10 \mathrm{~nm}$ have larger flux, and the effects of the Knudsen diffusion and slippage decrease with increasing throat radius. The variation of the non-dimensional apparent permeability with the pressure can thus not be determined using only the arithmetic average throat radius, but the flux distribution in the throats is also a significant factor.

S3Z and S1X were selected from Categories 1 and 2, respectively, for comparison of the flux-radius distributions under two extreme conditions, namely the Darcy condition and a pressure of 0.1 MPa, as shown in Fig. 6. Overall, the ratio of the flux of the throats with radii 


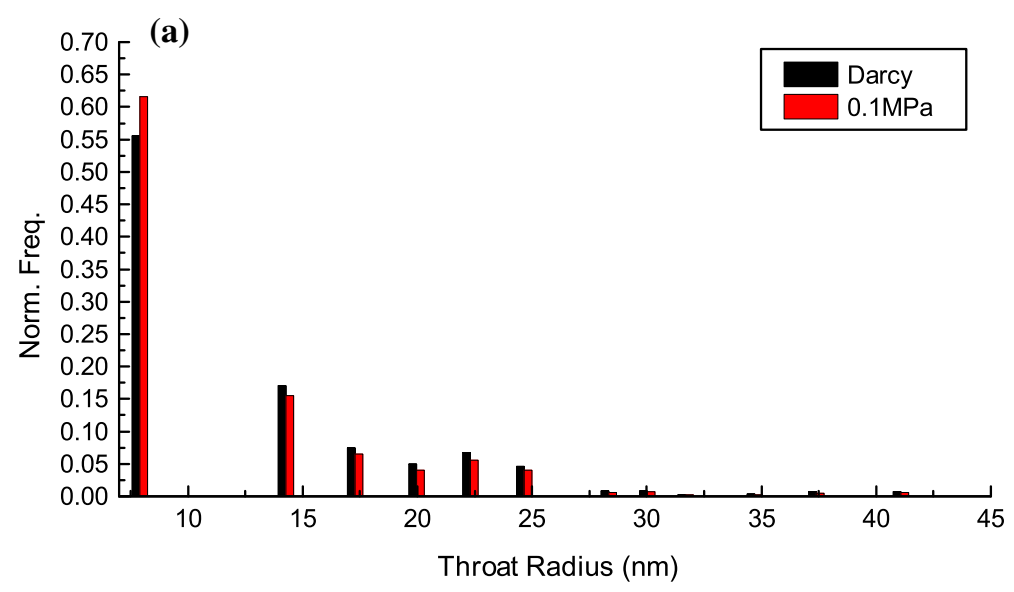

(b)

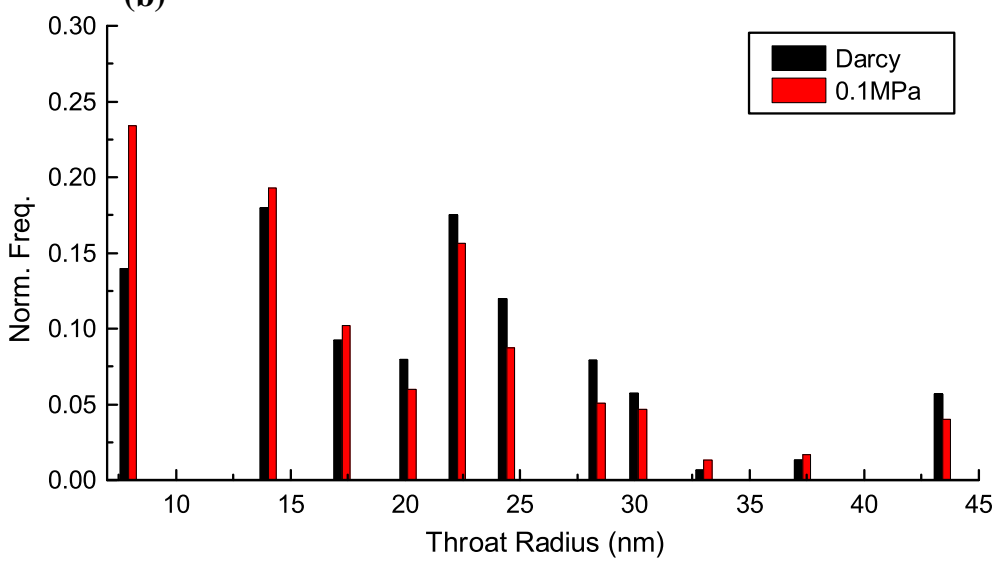

Fig. 6 Variation of the throat flux-radius distribution with pressure. a S3Z (Category 1), b S1X (Category 2)

smaller than the arithmetic average radius to the total flux of all the throats is larger when the Knudsen diffusion and slippage are taken into consideration ( $0.1 \mathrm{MPa}$ condition) compared to the Darcy condition, whereas the difference between samples is smaller. In addition, the flux ratio of the Category 2 samples changes more complexly between the $0.1 \mathrm{MPa}$ condition and the Darcy condition, indicating greater nonlinearity of the inner flow.

Tortuosity Tortuosity is defined as the average elongation of fluid paths and is one of the key parameters of the geometry and transport properties of porous media (Matyka and Koza 2012). Based on the results of simulations of flows through pore networks, the tortuosity is given by

$$
\tau=\frac{\sum_{i}\left|Q_{i}\right| \cdot\left|\vec{x}_{i 1}-\vec{x}_{i 2}\right|}{\left|\sum_{i} Q_{i} \cdot\left(\vec{x}_{i 1}-\vec{x}_{i 2}\right) \cdot \overrightarrow{I O}\right|}
$$

where $\overrightarrow{I O}$ is the unit vector in the direction of the pressure gradient, $Q i$ is the flux of pore throat $i, \vec{x}_{i 1}$ and $\vec{x}_{i 2}$ are the respective vectors of the pores adjacent to pore throat $i$, and $\mid$ | is the magnitude operator of the vectors. The elongations of the fluid paths in all the throats 


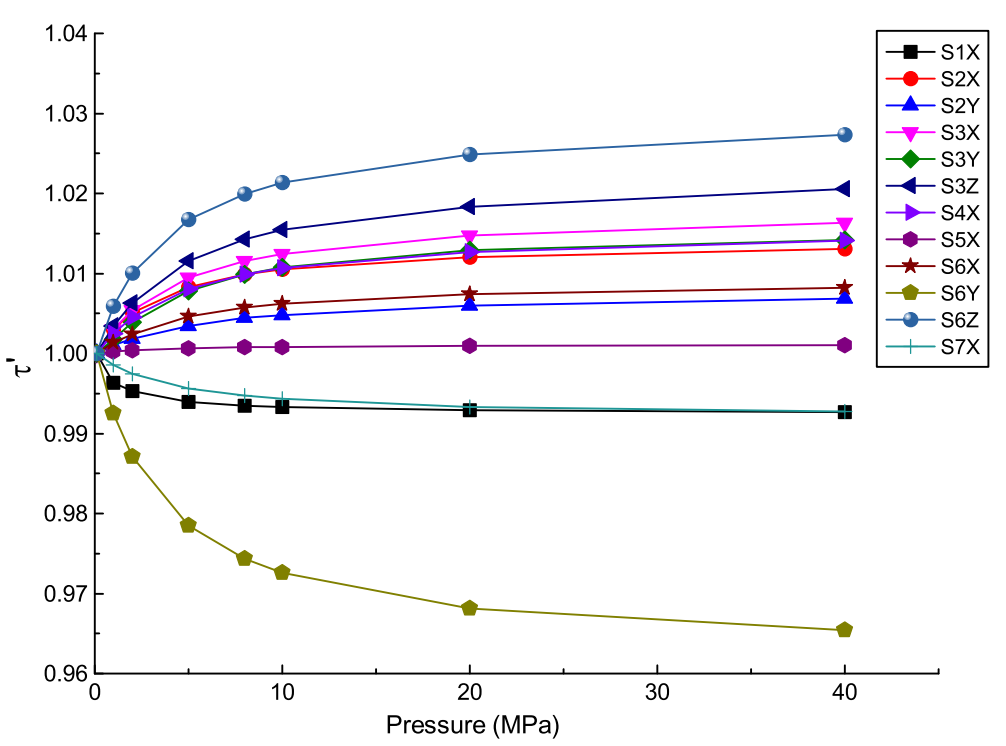

Fig. 7 Variation of the tortuosity with pressure

in the pore network are averaged using the respective flux magnitudes as the weighting coefficients.

Table 1 gives the determined tortuosities of all the pore clusters for Darcy flow. The values can be observed to range between 1.32 and 2.67, which is reasonable considering typical tortuosity values of 1.3 and 3 (Clennell 1997). The tortuosities in different directions of a given sample are also liable to differ significantly, due to the differing flow paths and flux distributions in the different directions.

When Knudsen diffusion and slippage are considered, the gas flow in the pore networks changes, including the tortuosity. Figure 7 shows the variation of the normalised tortuosity tau', which is the tortuosity under a certain pressure relative to that under a pressure of $0.1 \mathrm{MPa}$, with the pressure. The tortuosities of the Category 1 samples increase with increasing pressure, while the reverse is the case for the Category 2 samples, with the exception of S6X. The variation of the throat flux distribution with pressure can be used to explain this observation. When the pressure increases, the non-Darcy effect weakens and the flux ratios of the smaller throats decrease (red bars versus black bars, Fig. 6). It is apparent from the definition of tortuosity that the parameter is mainly controlled by the throats with larger fluxes. In the Category 1 samples, the radii of the controlling throats are smaller, and the fact that a decrease of the flux ratios of the controlling throats increases the tortuosity implies that the flow paths in the larger throats are more tortuous. Under a different condition, the radii of the controlling throats would be larger, and increasing the flux ratios of the controlling throats would decrease the tortuosity, indicating that the flow paths in the larger throats are less tortuous than those in the smaller throats. Using an artificial randomly generated pore network model, Zhang et al. (2014b) found that the tortuosity increased with increasing pressure. The results of the present simulations show that the two trends mentioned above occur in shale pore clusters. 


\section{Parameter Determination for the Effective Model of Pore Clusters}

In addition to the pore-scale simulation of the shale gas flow, there is another method that can be used to describe the gas transport. This other method involves the use of an effective model in which the complex pore networks (Fig. 1c) are represented by tubes (Fig. 1d). This model takes into consideration the apparent permeability $K_{\text {app }}$ and can be used to obtain an explicit expression that relates the parameter with other parameters such as the porosity, tortuosity, and $K_{d}$. For example, Darabi et al. (2012) proposed the following apparent permeability equation, which expresses $K_{\text {app }}$ in terms of the pore surface fractal dimension $D_{f}$, tortuosity $\tau$, and porosity $\phi$, which are three parameters related to the pore size $R_{\mathrm{avg}}, d_{p}, R_{\mathrm{nt}}$, and the absolute permeability $K_{d}$ :

$$
K_{\mathrm{app}}=\frac{2 R_{\mathrm{nt}} \mu_{g}}{3 \rho_{\mathrm{avg}}} \frac{\phi}{\tau}\left(\frac{d_{m}}{d_{p}}\right)^{D_{f}-2} \sqrt{\frac{8 M}{10^{3} \pi R T}}+K_{d}\left(1+\sqrt{\frac{8 \cdot 10^{3} \pi R T}{M}} \frac{\mu_{g}}{R_{\mathrm{avg}} p}\left(\frac{2}{\alpha}-1\right)\right)
$$

The above equation is, however, more like just a framework for the development of effective models because the included parameters are underdetermined and may have multiple values. It is therefore necessary to address these issues to obtain a more realistic effective model. The pore-scale modelling of shale gas flow through pore clusters can be used to determine both the characteristic parameters and $K_{\text {app }}$, and also affords a convenient means of distinguishing the parameters and establishing the relationships among them.

We here consider a scenario of the application of an effective model. Conventionally, pressure pulse-decay experiments are used to determine $K_{\text {app }}$ for given values of $T$ and $p$, based on which the value of $K_{\text {app }}$ for the actual formation conditions is then calculated. The calculation primarily involves the use of an effective model. To ensure correct calculation, an effective model and related parameters that are close to reality should be employed. When using Darabi et al. (2012)'s promising framework equation, the key parameters are those that are independent of $p$ and $T$, including $D_{f}, \tau, \phi, R_{\mathrm{avg}}, d_{p}, R_{\mathrm{nt}}$ and $K_{d}$. Among them, $D_{f}$ and $\tau$ can be determined by experiments or the methods used in the present study; there are several methods for determining $\phi, R_{\mathrm{avg}}, d_{p}$ and $R_{\mathrm{nt}}$, despite the existence of multiple definitions. Considering the smallness of shale throats, on the order of nanometres, the absolute permeabilities are ultra-low and difficult to measure steadily. The accurate determination of $K_{d}$ when only the measurement of $K_{\text {app }}$ is feasible is thus the key issue of this method. In this regard, Eq. (7) can be rewritten as follows:

$$
K_{d_{\mathrm{eff}}}=\frac{K_{\mathrm{app}}-\frac{2 R_{\mathrm{nt}} \mu_{g} \phi}{3 \rho_{\mathrm{avg}} \tau}\left(\frac{d_{m}}{d_{p}}\right)^{D_{f}-2} \sqrt{\frac{8 M}{10^{3} \pi R T}}}{1+\sqrt{\frac{8 \cdot 10^{3} \pi R T}{M}} \frac{\mu_{g}}{R_{\mathrm{avg}} p}\left(\frac{2}{\alpha}-1\right)}
$$

In this scenario, $K_{\text {app }}, p$, and $T$ are known, but $D_{f}$ and $\tau$ need to be determined. The choices among the multiple definitions of $\phi, R_{\mathrm{avg}}, d_{p}$, and $R_{\mathrm{nt}}$ are made based on whether $K_{d}$ is close to the intrinsic value for the sample. The different schemes used to determine these parameters require evaluation, and this can be done using the aforementioned pore-scale modelling, which enables the calculation of all the above parameters.

The porosity $\phi$ may be either the effective porosity $\phi_{e}$ or the flowing porosity $\phi_{f}$. In addition, $R_{\mathrm{avg}}, d_{p}$, and $R_{\mathrm{nt}}$ may be assumed to be equal and approximated by $\sqrt{8 K_{d}}$, with the assumption that the average throat radius is directly related to the absolute permeability. 
Table 2 Schemes for the effective model

\begin{tabular}{|c|c|c|c|c|c|}
\hline Scheme & $\phi$ & $R_{\text {avg }}$ & $d_{p}$ & $R_{\mathrm{nt}}$ & \\
\hline 1 & $\phi_{e}$ & $\sqrt{8 K_{d_{\mathrm{eff}}}}$ & $2 R_{\mathrm{avg}}$ & $\sqrt{8 K_{d_{\mathrm{eff}}}}$ & \multirow{4}{*}{$\frac{1}{D_{f}-3}$} \\
\hline 2 & $\phi_{f}$ & $\sqrt{\frac{8 \tau K_{d_{\mathrm{eff}}}}{\phi_{f}}}$ & $2 R_{\text {avg }}$ & $\sqrt{\frac{8 \tau K_{d_{\mathrm{eff}}}}{\phi_{f}}}$ & \\
\hline & & 1 & & $\Gamma$ & \\
\hline 3 & $\phi_{f}$ & $\overline{\frac{1}{N} \sum_{i=1}^{N} \frac{1}{r_{i}}}$ & $2 R_{\text {avg }}$ & $\overline{\frac{1}{N} \sum_{i=1}^{N}{\frac{1}{r_{i}}}^{D_{f-3}}}$ & \\
\hline
\end{tabular}

Alternatively, these parameters can be obtained as the respective average values from the throat radius distribution. The three possible schemes are as enumerated in Table 2. The schemes were applied to the 12 samples of this study, with Eq. (8) used to calculate $K_{d_{\text {eff }}}$. The relative error $\delta_{i}$ between $K_{d_{\text {eff }}}$ and $K_{d_{\mathrm{AB}}}$ for the $i$ th sample, based on its pore network model, was determined using

$$
\delta_{i}=\left|\frac{K_{d_{\mathrm{eff}}}^{i}}{K_{d_{\mathrm{AB}}}^{i}}-1\right|
$$

Because the non-Darcy effect was more obvious for a small average pressure, we first discuss the results for a pressure of $0.1 \mathrm{MPa}$.

Scheme 1 This scheme requires the distribution of the throat radius and the flow features. The effective porosity $\phi_{e}$ can be measured experimentally, and $R_{\mathrm{avg}}$ and $R_{\mathrm{nt}}$ are directly related to $K_{d_{\text {eff }}}$ and can be omitted from the equation. Minimum additional data are required for the computation. It can, however, be seen from Table 3 that the errors of this scheme are very large. To investigate the reason for this, we calculated the reverse values of $R_{\text {avg }}$ with respect to $K_{d_{\text {eff }}}$. The reverse values were determined to be within $0.01-0.41 \mathrm{~nm}$, which is obviously smaller than the arithmetic average of $R_{\mathrm{avg}, 1}$, namely, about $10 \mathrm{~nm}$.

Scheme 2 Considering the existence of a large number of blind ends in the pore network, and the fact that not all the throats contribute to the gas flow, we used the flow porosity $\phi_{f}$ rather than the effective porosity $\phi_{e}$. The employed equations of $R_{\text {avg }}$ and $R_{\mathrm{nt}}$ also take into consideration the effects of the porosity $\phi_{f}$ and tortuosity $\tau$. Consequently, Scheme 2 requires the flux data obtained by the PNM and more additional data than Scheme 1. However, as can be seen from Table 3, its results are better than those of Scheme 1, with the errors for some of the samples close to $30 \%$. The errors are also dispersed, with the reverse values of $R_{\mathrm{avg}, 2}$ being within $4.84-173.05 \mathrm{~nm}$, although most of them still significantly differ from the real values, resulting in large discrepancies.

Scheme 3 The difference between Schemes 2 and 3 is that $R_{\text {avg }}$ is independent of $K_{d_{\text {eff }}}$ in the latter, which alternatively uses the throat number-radius distribution data to generate different average values of $R_{\mathrm{avg}}$ and $R_{\mathrm{nt}}$, respectively. This approach considers that the powers of $R_{\mathrm{avg}}$ and $R_{\mathrm{nt}}$ are different and therefore does not represent them by a simple arithmetic average. This scheme requires the master flux data and radius distribution of the pore networks. The effective radii are basically about $10 \mathrm{~nm}$, which is close to the arithmetic average and quite reasonable. The maximum, minimum, and average relative errors of the results are 72.83 , 3.31 , and $19.51 \%$. This represents a significant improvement on the two previous schemes. The relative errors of most of the samples are less than 20\%, except for S1X, S5X, and S7X. 
Table 3 Comparison of the results of the different schemes

\begin{tabular}{|c|c|c|c|c|c|c|c|c|}
\hline Samples & $\begin{array}{l}R_{\mathrm{avg}, 1} \\
(\mathrm{~nm})\end{array}$ & $\begin{array}{l}R_{\mathrm{avg}, 2} \\
(\mathrm{~nm})\end{array}$ & $\begin{array}{l}R_{\mathrm{nt}, 3} \\
(\mathrm{~nm})\end{array}$ & $\begin{array}{l}R_{\mathrm{avg}, 3} \\
(\mathrm{~nm})\end{array}$ & $\begin{array}{l}K_{d_{\mathrm{AB}}} \\
(\mathrm{nD})\end{array}$ & $\begin{array}{l}K_{d_{\mathrm{eff}, 1}} \\
(\mathrm{nD})\end{array}$ & $\begin{array}{l}K_{d_{\mathrm{eff}, 2}} \\
(\mathrm{nD})\end{array}$ & $\begin{array}{l}K_{d_{\text {eff }, 3}} \\
(\mathrm{nD})\end{array}$ \\
\hline S1X & 0.41 & 150.02 & 10.47 & 9.37 & 1445.39 & 88.10 & 11787.75 & 998.85 \\
\hline $\mathrm{S} 2 \mathrm{X}$ & 0.02 & 32.59 & 8.83 & 8.37 & 32.19 & 0.18 & 130.73 & 38.18 \\
\hline $\mathrm{S} 2 \mathrm{Y}$ & 0.01 & 10.60 & 8.82 & 8.37 & 14.51 & 0.04 & 19.25 & 15.53 \\
\hline S3X & 0.02 & 23.12 & 8.91 & 8.39 & 35.79 & 0.21 & 102.56 & 41.24 \\
\hline S3Y & 0.01 & 6.05 & 8.96 & 8.42 & 7.38 & 0.01 & 5.17 & 6.67 \\
\hline S3Z & 0.01 & 13.68 & 8.95 & 8.42 & 13.61 & 0.03 & 22.99 & 15.03 \\
\hline S4X & 0.01 & 13.68 & 8.46 & 8.19 & 17.47 & 0.05 & 31.21 & 19.74 \\
\hline S5X & 0.02 & 51.74 & 9.98 & 8.87 & 18.58 & 0.12 & 128.44 & 32.11 \\
\hline S6X & 0.06 & 68.63 & 9.81 & 8.85 & 121.47 & 1.68 & 779.45 & 125.49 \\
\hline S6Y & 0.04 & 44.58 & 9.81 & 8.86 & 85.99 & 0.64 & 321.77 & 75.92 \\
\hline S6Z & 0.01 & 4.84 & 9.73 & 8.82 & 4.97 & 0.01 & 2.92 & 4.13 \\
\hline S7X & 0.15 & 173.05 & 13.13 & 11.29 & 330.97 & 10.84 & 4034.58 & 411.99 \\
\hline \multicolumn{6}{|c|}{ Maximum relative error } & $99.86 \%$ & $1119 \%$ & $72.83 \%$ \\
\hline \multicolumn{6}{|c|}{ Minimum relative error } & $93.90 \%$ & $29.99 \%$ & $3.31 \%$ \\
\hline \multicolumn{6}{|c|}{ Average relative error } & $98.80 \%$ & $332.17 \%$ & $19.51 \%$ \\
\hline
\end{tabular}

The numbers in subscript represent the scheme index

Excluding these three samples, the overall average relative error reduces to $14.65 \%$, which is quite acceptable. This shows that the scheme can be used to obtain relatively accurate absolute permeabilities for most samples. The typical features of S1X, S5X, and S7X, for which the errors exceed 20\%, are much fewer pores and throats. This implies that the scheme may not be sufficiently accurate for less-developed pore networks.

A comprehensive analysis of the above three schemes revealed that they require accurate values of $\phi, R_{\mathrm{avg}}$ and $R_{\mathrm{nt}}$. Figure 8 shows the variation of the Scheme 3 relative error $\delta_{i}$ with the pressure for all the samples. With the exception of the three samples with less-developed pore networks, the errors are all less than $20 \%$ and generally decrease with increasing pressure.

Having determined the best parameter scheme, the following forward expression of the effective model for shale gas flow from the pore scale to the cluster scale was obtained:

$$
\left\{\begin{array}{l}
K_{\mathrm{app}}=\frac{2 R_{\mathrm{nt}} \mu_{g} \phi_{f}}{3 \rho_{\mathrm{avg}} \tau}\left(\frac{d_{m}}{2 R_{\mathrm{nt}}}\right)^{D_{f}-2} \sqrt{\frac{8 M}{10^{3} \pi R T}}+K_{d}\left(1+\sqrt{\frac{8 \cdot 10^{3} \pi R T}{M}} \frac{\mu_{g}}{R_{\mathrm{avg}} p}\left(\frac{2}{\alpha}-1\right)\right) \\
R_{\mathrm{nt}}=\left[\frac{1}{\frac{1}{N} \sum_{i=1}^{N}{\frac{1}{r_{i}}}^{D_{f}-3}}\right]^{\frac{1}{D_{f}-3}} \\
R_{\mathrm{avg}}=\frac{1}{\frac{1}{N} \sum_{i=1}^{N} \frac{1}{r_{i}}}
\end{array}\right.
$$

The model takes its framework from Darabi et al. (2012)s equation. However, its parameters are more clearly defined by the pore network model. A detailed process for determining some of the parameters is presented in the flow chart in Fig. 9. The process can also be applied to 


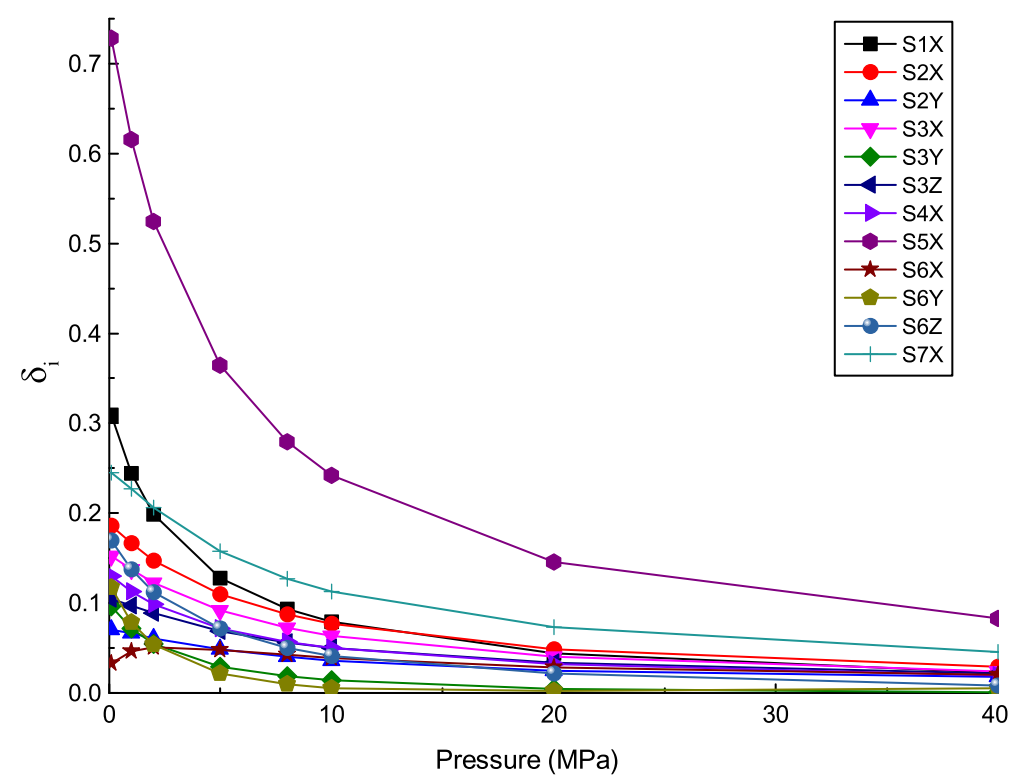

Fig. 8 Variation of the Scheme 3 relative error with the pressure for all the samples

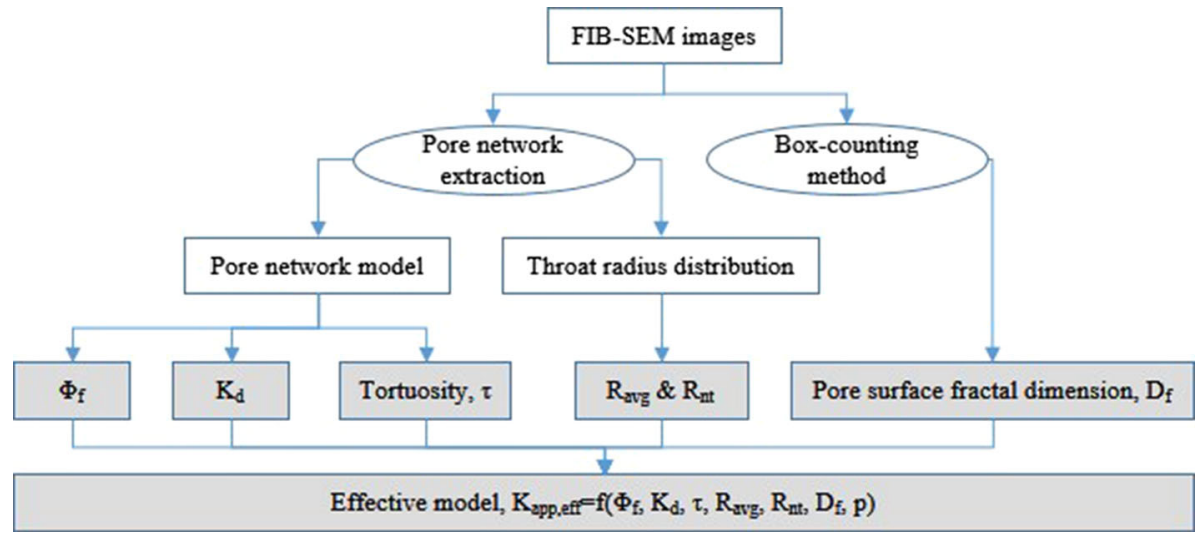

Fig. 9 Process flow chart for developing an effective model from the pore scale to the cluster scale

other flow models such as those that consider adsorption/desorption (Zamirian et al. 2014; Wasaki and Akkutlu 2015; Jin and Firoozabadi 2015; Kazemi and Takbiri-Borujeni 2016), provided that their frameworks are known. The process can also be used to develop an original effective model.

\section{Conclusion}

Nanometre-resolution 3D FIB-SEM images and a high-precision pore network extraction algorithm (AB algorithm) were used to extract the pore networks of seven shale pore clusters. Considering the effects of the pore surface fractal dimension, Knudsen diffusion, and 
slippage, the apparent permeabilities of the pore networks for shale gas were calculated and the related characteristic parameters were analysed. The best parameter scheme for developing an effective model was identified from among three schemes based on the pore-scale modelling results, and an effective model for upscaling between the pores and the clusters was finally developed. Following are the conclusions drawn from the study:

(1) The AB algorithm is effective for extracting the nano-scale pore network in shale. In addition, structural parameters such as the flow porosity, coordination number distribution, throat number-radius distribution, throat flux-radius distribution, and tortuosity can be determined by pore network modelling. There are, however, some pore throats with zero flux and many dead-end pores. The tortuosities of several of the samples considered in this study increased with increasing pressure, while the reverse was the case for others.

(2) Although the throat radius distributions of the considered samples are very similar, the samples can be divided into two categories based on the throat flux-radius distributions. Category 1 comprises samples for which the ratio of the fluxes of the throats with radii smaller than the average radius to the total flux exceeds $50 \%$, while Category 2 comprises those for which the ratio is less than 50\%. Samples of the same cluster but with different directional properties may belong to different categories. In addition, the throat flux distribution varies with the pressure, with the flux ratio of the smaller throats tending to decrease with increasing pressure.

(3) An effective model that relates the absolute permeability, apparent permeability, and other characteristic parameters was developed. Three parameter schemes based on the pore network model results were considered, and their relative errors were evaluated. The first two schemes, which relate $K_{d}$ and $R_{\text {avg }}$, were observed to have larger relative errors, while the third scheme, which considers the throat number-radius distribution and the flow porosity, was found to be the most accurate

Acknowledgements This work is supported by the Strategic Priority Research Program of the Chinese Academy of Sciences (Grant No. XDB10020302), the National Natural Science Foundation of China (Grant No. 41574129 and 41690132), the National Program on Key Basic Research Project (973 Program, Grant No. 2014CB239004), the National Science and Technology Major Project of the Ministry of Science and Technology of China and the China Geological Survey Project (Grant No. 12120114055801).

\section{References}

Agrawal, A., Prabhu, S.V.: Survey on measurement of tangential momentum accommodation coefficient. J. Vac. Sci. Technol. A (2008). doi:10.1116/1.2943641

Ai, T., Zhang, R., Zhou, H.W., Pei, J.L.: Box-counting methods to directly estimate the fractal dimension of a rock surface. Appl. Surf. Sci. (2014). doi:10.1016/j.apsusc.2014.06.152

Akkutlu, I.Y., Fathi, E.: Multiscale gas transport in shales with local kerogen heterogeneities. SPE J. (2012). doi:10.2118/146422-PA

Chen, C., Hu, D.D., Westacott, D., Loveless, D.: Nanometer-scale characterization of microscopic pores in shale kerogen by image analysis and pore-scale modeling. Geochem. Geophys. Geosyst. (2013). doi:10. 1002/ggge.20254

Chen, L., Zhang, L., Kang, Q.J., Yao, J., Tao, W.Q.: Nanoscale simulation of shale transport properties using the lattice Boltzmann method: permeability and diffusivity. Sci. Rep. (2015). doi:10.1038/srep08089

Civan, F.: Scale effect on porosity and permeability-kinetics, model, and correlation. AIChE J. (2001). doi:10. 1002/aic.690470206

Civan, F.: Relating permeability to pore connectivity using a power-law flow unit equation. Petrophys. J. 43(6), 457-476 (2002) 
Civan, F.: Effective correlation of apparent gas permeability in tight porous media. Transp. Porous Media (2010). doi:10.1007/s11242-009-9432-z

Civan F.: Improved permeability prediction for heterogeneous porous media by bundle-of-leaky-tubes with cross-flow model. In: Proceedings (CD) of the 5th International Conference on Porous Media and Its Applications in Science, Engineering and Industry (ICPMV) (2014)

Clennell, M.B.: Tortuosity: A Guide Through the Maze. Geological Society, Special Publications, London (1997). doi:10.1144/GSL.SP.1997.122.01.18

Coppens, M.O.: The effect of fractal surface roughness on diffusion and reaction in porous catalysts-from fundamentals to practical applications. Catal. Today (1999). doi:10.1016/S0920-5861(99)00118-2

Darabi, H., Ettehad, A., Javadpour, F., Sepehrnoori, K.: Gas flow in ultra-tight shale strata. J. Fluid Mech. (2012). doi:10.1017/jfm.2012.424

Deng, J., Zhu, W.Y., Ma, Q.: A new seepage model for shale gas reservoir and productivity analysis of fractured well. Fuel (2014). doi:10.1016/j.fuel.2014.02.001

Dong, H., Blunt, M.J.: Pore-network extraction from micro-computerized-tomography images. Phys. Rev. E (2009). doi:10.1103/PhysRevE.80.036307

Fathi, E., Akkutlu, I.Y.: Lattice Boltzmann method for simulation of shale gas transport in Kerogen. SPE J. (2012). doi:10.2118/146821-PA

Florence, F.A., Rushing, J.A., Newsham, K.E., Blasingame, T.A.: Improved permeability prediction relations for low permeability sands. In: Rocky Mountain Oil, Gas Technology Symposium (2007). doi:10.2118/ 107954-MS

Guo, C.H., Bai, B.J., Wei, M.Z., He, X.M., Wu, Y.S.: Study on gas flow in nano pores of shale gas reservoir. In: SPE Unconventional Resources Conference Canada (2013). doi:10.2118/167179-MS

Harpreet, S., Javadpour, F.: Langmuir slip-Langmuir sorption permeability model of shale. Fuel (2016). doi:10. 1016/j.fuel.2015.09.073

Javadpour, F., Fisher, D., Unsworth, M.: Nanoscale gas flow in shale gas sediments. J. Can. Pet. Technol. (2007). doi:10.2118/07-10-06

Javadpour, F.: Nanopores and apparent permeability of gas flow in mudrocks (shales and siltstone). J. Can. Pet. Technol. (2009). doi:10.2118/09-08-16-DA

Jiang, Z., Wu, K., Couples, G., van Dijke, M.I.J., Sorbie, K.S., Ma, J.: Efficient extraction of networks from three-dimensional porous media. Water Resour. Res. (2007). doi:10.1029/2006WR005780

Jin, Z.H., Firoozabadi, A.: Flow of methane in shale nanopores at low and high pressure by molecular dynamics simulations. J. Chem. Phys. (2015). doi:10.1063/1.4930006

Kazemi, M., Takbiri-Borujeni, A.: An analytical model for shale gas permeability. Int. J. Coal Geol. (2015). doi:10.1016/j.coal.2015.05.010

Kazemi, M., Takbiri-Borujeni, A.: Non-equilibrium molecular dynamics simulation of gas flow in organic nanochannels. J. Nat. Gas Sci. Eng. (2016). doi:10.1016/j.jngse.2016.05.068

Lindquist, W.B., Lee, S.M., Coker, D.A., Jones, K.W., Spanne, P.: Medial axis analysis of void structure in three-dimensional tomographic images of porous media. J. Geophys. Res. Solid Earth (1996). doi:10. 1029/95JB03039

Lunati, I., Lee, S.H.A.: A dual-tube model for gas dynamics in fractured nanoporous shale formations. J. Fluid Mech. (2014). doi:10.1017/jfm.2014.519

Ma, J.S., Sanchez, J.P., Wu, K.J., Couples, G.D., Jiang, Z.Y.: A pore network model for simulating non-ideal gas flow in micro- and nano-porous materials. Fuel (2014). doi:10.1016/j.fuel.2013.08.041

Matyka, M., Koza, Z.: How to calculate tortuosity easily? In: AIP Conference Proceedings (2012). doi:10. $1063 / 1.4711147$

Mehmani, A., Prodanovic, M., Javadpour, F.: Multiscale, multiphysics network modeling of shale matrix gas flows. Transp. Porous Media (2013). doi:10.1007/s11242-013-0191-5

Naraghi, M.E., Javadpour, F.: A stochastic permeability model for the shale-gas systems. Int. J. Coal Geol. (2015). doi:10.1016/j.coal.2015.02.004

Okabe, H., Blunt, M.J.: Prediction of permeability for porous media reconstructed using multiple-point statistics. Phys. Rev. E (2004). doi:10.1103/PhysRevE.70.066135

Pan, C.X., Luo, L.S., Miller, C.T.: An evaluation of lattice Boltzmann schemes for porous medium flow simulation. Comput. Fluids (2006). doi:10.1016/j.compfluid.2005.03.008

Roy, S., Raju, R., Chuang, H.F., Cruden, B.A., Meyyappan, M.: Modeling gas flow through microchannels and nanopores. J. Appl. Phys. (2003). doi:10.1063/1.1559936

Schneider, C.A., Rasband, W.S., Eliceiri, K.W.: NIH image to imageJ: 25 years of image analysis. Nat. Methods (2012). doi:10.1038/nmeth.2089

Swami, V., Settari, A.: A pore scale gas flow model for shale gas reservoir. In: SPE Americas Unconventional Resources Conference (2012). doi:10.2118/155756-MS 
Swami, V., Settari, A., Javadpour, F.: A numerical model for multi-mechanism flow in shale gas reservoirs with application to laboratory scale testing. In: EAGE Annual Conference, Exhibition incorporating SPE Europec (2013). doi:10.2118/164840-MS

Tahmasebi, P., Javadpour, F., Sahimi, M.: Three-dimensional stochastic characterization of shale SEM images (2015). doi:10.1007/s11242-015-0570-1

Wasaki, A., Akkutlu, I.Y.: Permeability of organic-rich shale. SPE J. (2015). doi:10.2118/170830-PA

Zhang, X., Xiao, L., Shan, X., Guo, L.: Lattice Boltzmann simulation of shale gas transport in organic nanopores. Sci. Rep. (2014a). doi:10.1038/srep04843

Zhang, Z.B., Lin, M., Li, X.: Characteristics of tortuosity in tight oil reservoirs. Bull. Mineral. Petrol. Geochem. (2014b). doi:10.3969/j.issn.1007-2802.2015.01.006

Zamirian, M., Aminian, K., Fathi, E., Ameri, S.: A fast and robust technique for accurate measurement of the organic-rich shales characteristics under steady-state conditions. In: SPE Eastern Regional Meeting (2014). doi:10.2118/171018-MS.1007-2802.2015.01.006 